\title{
Influence of the ICDAS E-Learning Program for Occlusal Caries Detection on Dental Students
}

\author{
Michele B. Diniz, D.D.S., M.S.D.; Luciana Monti Lima, D.D.S., M.S.D., Ph.D.; Lourdes \\ Santos-Pinto, D.D.S., M.S.D., Ph.D.; George J. Eckert, M.A.S.; Andrea G. Ferreira \\ Zandoná, D.D.S., M.S.D., Ph.D.; Rita de Cássia Loiola Cordeiro, D.D.S., M.S.D., Ph.D.
}

Abstract: The aim of this study was to evaluate the influence of ICDAS training in a group of dental students for occlusal caries detection in permanent teeth. Premolars and molars $(\mathrm{N}=104)$ with occlusal surfaces varying from ICDAS scores 0 to 6 were cleaned, one occlusal site per tooth was selected, and a photograph taken to identify the site. Eight senior dental students examined the teeth twice with a one-week interval between examinations during each of two phases: before and after the ICDAS e-learning program. Teeth were histologically assessed for caries extension. Intraclass correlation coefficients for intra- and interexaminer repeatability were high, both before ( 0.75 and 0.72 , respectively) and after e-learning $(0.82$ and 0.78 , respectively). The ICDAS scores decreased significantly from before to after e-learning $(\mathrm{p}=0.0001)$. Correlation between ICDAS scores and histology scores was moderate ( 0.57 before e-learning and 0.61 after). Although the ROC curve shows an improvement in the use of the ICDAS scoring after e-learning, the difference was not significant $(\mathrm{p}=0.10)$. Specificity of the ICDAS scores significantly improved after e-learning ( 77 percent vs. 36 percent), and sensitivity was reduced slightly after e-learning (87 percent vs. 92 percent). The ICDAS e-learning program improved the performance of the diagnostic skills of the investigated students for the detection of occlusal caries lesions.

Dr. Diniz is a Ph.D. Student, Department of Pediatric Dentistry, Araraquara Dental School, Sao Paulo, Brazil; Dr. Lima is with the Department of Pediatric Dentistry, Araraquara Dental School, Sao Paulo, Brazil; Dr. Santos-Pinto is Associate Professor, Department of Pediatric Dentistry, Araraquara Dental School, Sao Paulo, Brazil; Mr. Eckert is Biostatistician Supervisor, Division of Biostatistics, School of Medicine, Indiana University; Dr. Zandoná is Associate Professor, Department of Preventive and Community Dentistry, School of Dentistry, Indiana University; and Dr. Cordeiro is Adjunct Professor, Department of Pediatric Dentistry, Araraquara Dental School, Sao Paulo, Brazil. Direct correspondence and requests for reprints to Dr. Michele B. Diniz, Department of Pediatric Dentistry, Araraquara Dental School, Rua Humaitá, 1680 Centro Araraquara, Sao Paulo 14801-903, Brazil; 55-16-3301-6331 phone; 55-16-3301-6329 fax; mibdiniz@hotmail.com.

Keywords: dental caries, educational methodologies, oral diagnosis, dental education

Submitted for publication 11/8/09; accepted 2/9/10

$\mathrm{S}$ everal clinical caries detection criteria describing various stages of dental caries have been developed. ${ }^{1,2}$ However, many of these criteria are ambiguous in their descriptions and fail to include the earliest signs of disease. It is important to point out that many lesions are at the subclinical stages of development, which are very small to be detected by visual examination. ${ }^{3}$ In commonality, clinical caries criteria are based on visual inspection and on subjective assessments of color, translucence, and dental hardness, ${ }^{4,5}$ having high specificity and low sensitivity. ${ }^{6}$ Some criteria have been proposed to reduce the subjectivity and increase the sensitivity, providing defined descriptors of various severity stages of caries lesions. ${ }^{4,5,7}$
Recently, the International Caries Detection \& Assessment System (ICDAS), with new visual criteria, was developed. ${ }^{7}$ The ICDAS criteria rely on the visual inspection of clean, plaque-free wet and dried teeth. The system describes six stages of caries severity, varying from initial changes visible in enamel to frank cavitation in dentine. ${ }^{7-9}$ Some studies have shown good reproducibility and accuracy of ICDAS for occlusal caries detection in permanent teeth $^{7,9-12}$ and good reproducibility in epidemiological studies. ${ }^{13-15}$

The ICDAS e-learning program, developed by the ICDAS Foundation (distribution from Smile-On Limited), is a novel tool available by download via the Internet to explain the method and to provide an 
introduction of the criteria to novice users. It consists of a ninety-minute course divided into introduction, ICDAS examination protocol, ICDAS caries codes, how to apply the coding system, a decision tree to help with the codes, special considerations, and how to collect data for the recording codes. It also includes interactive quizzes.

To date, there are no published studies evaluating the impact of the ICDAS e-learning program on the ability of novices to use the criteria. The aim of this in vitro study was to evaluate the influence of this e-learning program to detect occlusal caries in permanent teeth on a group of dental students.

\section{Materials and Methods}

One hundred four permanent human premolars and molars, without sealants or restorations and with a range of ICDAS codes between 0 and 6 , were selected from a pool of extracted teeth, which were stored in 0.1 percent thymol solution at $4{ }^{\circ} \mathrm{C}$ until use. Calculus and debris were removed using a scaler, and the teeth were then cleaned for $15 \mathrm{~s}$ with water and Robson brush in a low-speed handpiece. Photographs of the occlusal surfaces were taken under a stereomicroscope (SZX7, Olympus Corporation, Tokyo, Japan) with magnification of 10x, and one occlusal site per tooth was selected (test site).

\section{Two Phases of the Study}

The study was conducted in two phases: before and after the e-learning program training. During the first phase, eight senior dental students from Araraquara Dentistry School, São Paulo State University, Brazil, were invited to participate as volunteers in this study. They were introduced to the ICDAS criteria by a group of supervisors who had previous experience with ICDAS. The details of each code were discussed based on a published paper concerning ICDAS ${ }^{10}$ until a consensus was reached. A two-hour session with everyone present was provided. The first hour was a lecture describing each code, and the next hour was a practical training session with twenty-five extracted teeth with different ICDAS codes, specifically selected for this purpose. After one week, the students had the same practical training session.

One week later, visual examination of the extracted teeth using the ICDAS criteria was performed, with repeat examinations performed after one week to remove bias associated by memorizing the ICDAS scores. The dental students were guided by black and white photographs printed in draft-quality paper containing a dot on the test site to allow the examiners to analyze precisely the same area. The specific site was hidden by the dot to avoid biasing the ICDAS exam by the photographic images. The teeth were examined in the same room with the aid of a light reflector and a three-in-one air syringe. First, they were analyzed moist and then dried, according to Ismail et al. ${ }^{7}$ and Jablonski-Momeni et al. ${ }^{10} \mathrm{~A}$ ball-ended explorer was used without applying pressure to confirm only cavitation. The teeth were coded as suggested by Jablonski-Momeni et al., ${ }^{10}$ ranging from first visible carious change in enamel to extensive cavitation.

The second phase took place after the e-learning program. The dental students simultaneously performed the online ICDAS e-learning in ninety minutes, with oversight by two supervisors, four weeks after the session introducing the criteria. After completion of the e-learning, there was an additional discussion session concerning the ICDAS codes. The students were allowed to go back and forth at their own pace during the discussion session. The students examined the same set of teeth a second time with a one-week interval between examinations, following the ICDAS criteria described by the e-learning program.

There are some differences between the ICDAS codes published by Jablonski-Momeni et al. ${ }^{10}$ and the codes proposed by the e-learning. In general, the ICDAS e-learning program explains and illustrates more precisely the codes with details of each condition. ICDAS codes are briefly described by the study cited above.

\section{Validation and Statistical Analysis}

The teeth were longitudinally sectioned through the center of each test site with a water-cooled diamond disk in a machine (ISOMET 1000, Buehler Ltd., Lake Bluff, IL, USA). The most severe section from each tooth was analyzed under a stereomicroscope with magnification of 10x. The deepest demineralization area either in the enamel or both the enamel and the dentin tissues were analyzed by two examiners according to the five-point histological classification system proposed by Ekstrand et al. ${ }^{4}$ to record caries extension at each tested site.

Intra- and interexaminer repeatability of the ICDAS severity scores was assessed using two-way tables and intraclass correlation coefficients (ICCs). ICCs were used rather than kappa statistics because 
of the full set of repeats for all specimens for all eight examiners.

Repeated measures ANOVA was used to test for a significant change in the ICDAS scores before and after the e-learning program. Comparisons between the percent correct, specificity, sensitivity, and area under the ROC curve of the ICDAS scores before versus after e-learning as well the correlation between the ICDAS scores and the histology scores were performed using bootstrap analyses. The bootstrap methodology uses resampling techniques to estimate statistics and perform comparisons for values that are not normally distributed. In this case, the bootstrap also provided a way to properly account for the correlations between examiners, between repeats, and between the before and after e-learning scores. Accuracy was measured by the area under the ROC curve, using the following guidelines: an area of 0.90 to 1.0 represents an excellent test; 0.80 to 0.90 is good; 0.70 to 0.80 is fair; 0.60 to 0.70 is poor; and 0.50 to 0.60 is a fail. ${ }^{16}$

\section{Results}

From the 104 occlusal test sites analyzed in this study, the histological examination showed that six of them had no caries, twenty-one had caries lesion limited to the outer half of the enamel, forty-seven had caries extending into the inner half of the enamel or outer half of the dentin, eight had caries limited to the middle third of the dentine, and twenty-two had caries involving the inner half of the dentine.

Intraclass correlation coefficients for intra- and interexaminer repeatability were high, indicating good repeatability for each examiner and between examiners, both before ( 0.75 and 0.72 , respectively) and after the e-learning program ( 0.82 and 0.78 , respectively). Overall, the intra- and interexaminer repeatability was

Table 1. Intraclass coefficients (ICCs) for inter- and intraexaminer repeatability of the ICDAS before and after the e-learning program

\begin{tabular}{lcc} 
& \multicolumn{2}{c}{ Repeatability ICCs } \\
\cline { 2 - 3 } Phase & Interexaminer & Intraexaminer \\
\hline Before e-learning & 0.72 & 0.75 \\
After e-learning & 0.78 & 0.82 \\
Note: No significant differences were observed between the \\
two phases.
\end{tabular}

slightly higher after the e-learning than before the elearning program, but were not significantly different $(p>0.05)$ based on the confidence intervals. Table 1 shows the intraclass coefficients (ICCs) for inter- and intraexaminer repeatability of the ICDAS before and after the e-learning program training.

Table 2 shows the cross-tabulation of the ICDAS scores before and after the e-learning program. The ICDAS scores decreased significantly from before the e-learning to after the e-learning program $(\mathrm{p}=0.0001)$. The average (SE) decrease in the scores was $0.68(0.08)$.

Specificity, sensitivity, area under the ROC curve, and correlation between ICDAS scores and histology are shown in Table 3. The correlation between the ICDAS scores and histology scores was moderate $(0.57$ before the e-learning and 0.61 after it). The specificity of the ICDAS scores was significantly improved after the e-learning program $(p=0.0001,0.77$ vs. 0.36). However, sensitivity was reduced slightly after the e-learning program $(\mathrm{p}=0.0061,0.87$ vs. 0.92$)$.

Figure 1 shows the ROC curve for the ICDAS before and after the e-learning program. Although the ROC curve shows an improvement in the use of the ICDAS scoring after the e-learning program, due to variability in the curves, the difference was not statistically significant $(\mathrm{p}=0.10)$.

\section{Discussion}

Various techniques of caries detection are taught to dental students during their dental education, including visual, tactile, and radiographic examination. ${ }^{17}$ However, the major challenge for the students is to detect caries lesions at an early stage, before invasive intervention is needed..$^{18}$ Since conventional visual examination usually has low sensitivity, examiners could miss many lesions present on occlusal surfaces. ${ }^{6}$ Recently, the ICDAS, a new visual criteria system, was developed to help the examiners detect the initial changes in dental surfaces due to caries development. In the literature there are some studies concerning the ICDAS for occlusal caries detection, indicating good reproducibility and accuracy. ${ }^{7,9,10}$ Most of these studies were performed by examiners who received extensive training, including in vivo exams before using the ICDAS criteria. An online e-learning program concerning the ICDAS criteria was recently introduced to promote examiners' training. 
Table 2. Cross-tabulation showing the ICDAS scores before and after the e-learning program

\begin{tabular}{|c|c|c|c|c|c|c|c|c|}
\hline \multirow[t]{2}{*}{$\begin{array}{l}\text { ICDAS before the } \\
\text { e-learning program }\end{array}$} & \multicolumn{8}{|c|}{ ICDAS after the e-learning program } \\
\hline & 0 & 1 & 2 & 3 & 4 & 5 & 6 & Total \\
\hline 0 & 85 & 49 & 15 & 8 & 1 & 0 & 1 & 159 \\
\hline 1 & 51 & 55 & 37 & 6 & 0 & 0 & 0 & 149 \\
\hline 2 & 28 & 64 & 76 & 16 & 6 & 1 & 0 & 191 \\
\hline 3 & 110 & 210 & 223 & 258 & 16 & 13 & 3 & 833 \\
\hline 4 & 0 & 2 & 2 & 1 & 17 & 1 & 0 & 23 \\
\hline 5 & 0 & 2 & 7 & 33 & 10 & 93 & 2 & 147 \\
\hline 6 & 1 & 1 & 0 & 2 & 0 & 99 & 59 & 162 \\
\hline Total & 275 & 383 & 360 & 324 & 50 & 207 & 65 & 1,664 \\
\hline
\end{tabular}

Table 3. Specificity, sensitivity, area under the ROC curve $\left(A_{z}\right)$, and correlation of the ICDAS criteria

\begin{tabular}{lcccc} 
Phase & Specificity & Sensitivity & $\mathrm{A}_{z}$ & Correlation \\
\hline Before e-learning & $0.36^{\mathrm{a}}$ & $0.92^{\mathrm{a}}$ & $0.80^{\mathrm{a}}$ & $0.57^{\mathrm{a}}$ \\
After e-learning & $0.77^{\mathrm{b}}$ & $0.87^{\mathrm{b}}$ & $0.88^{\mathrm{a}}$ & $0.61^{\mathrm{a}}$
\end{tabular}

Note: Different superscript letters in the same column represent significant differences $(p<0.05)$.

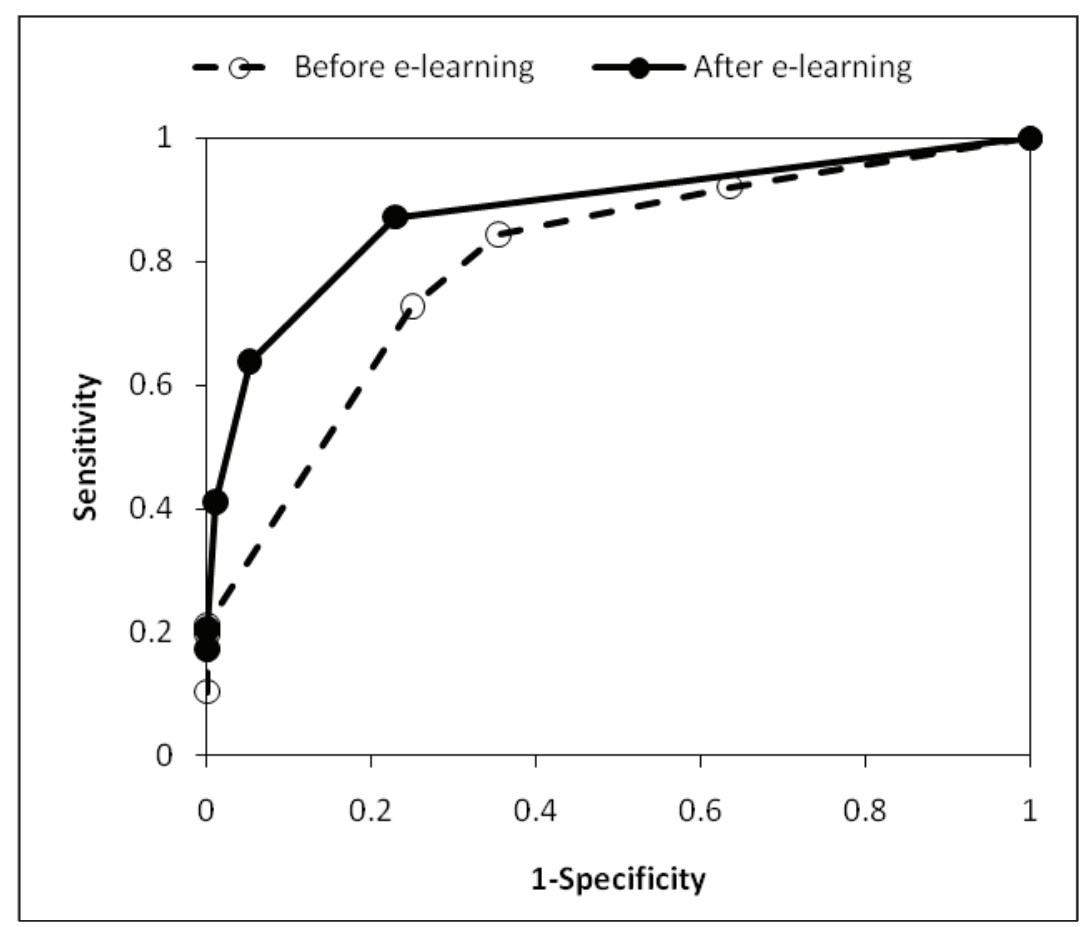

Figure 1. ROC curve for the ICDAS before and after the e-learning program 
This study evaluated the performance of dental students on the use of ICDAS to detect occlusal caries lesions in permanent teeth before and after the training-learning. Regarding reliability, although the most common measure used in dental epidemiology is the kappa coefficient, ${ }^{4,19}$ in this study the correlation between observers was calculated with a variance components analysis using the intraclass correlation coefficient (ICC). ${ }^{20}$ The ICC measures the strength of agreement among observers and has a maximum of 1 when agreement is perfect. However, it is important to mention that ICC does not rule out bias from memorizing individual sample teeth. According to Ongkosuwito et al., ${ }^{21}$ the ICC is equivalent to a weighted kappa coefficient, an ICC value of 0.61 to 0.80 is interpreted as substantial agreement, and an ICC of 0.81 to 1.00 is an almost perfect agreement. In this study, the intraclass coefficients were high, varying from 0.72 to 0.82 for intra- and interexaminer repeatability in both phases, indicating substantial to excellent agreement. According to Ismail et al., ${ }^{7}$ in an in vivo study, the ICDAS presents good to excellent reproducibility, even when applied by examiners who have no previous experience in epidemiological dental examination. They showed higher weighted kappa values for intraexaminer (varying from 0.59 to 0.79 ) and interexaminer reproducibility (varying from 0.63 to 0.75 ). Jablonski-Momeni et al., ${ }^{10}$ in an in vitro study, presented weighted kappa values ranging from 0.62 to 0.82 for interexaminer repeatability and from 0.74 to 0.83 for intraexaminer repeatability. Similar kappa values were also found by Ekstrand et al. ${ }^{9}$ for intra- $(0.82$ to 0.87$)$ and interexaminer agreement (0.82). Kühnisch et al., ${ }^{15}$ in an epidemiological study, showed high values of weighted kappa for both intra- (0.88) and interexaminer (0.90) repeatability.

Weighted kappas can vary between studies due to the schema used for weighting the differences, with absolute and square differences the most commonly used. Since most papers do not indicate the weighting scheme used when computing weighted kappas, variation between studies may be due simply to computing differences and not differences in the actual level of agreement. The reproducibility achieved in this study is satisfactory when compared with those cited above, even though it could be observed that most of the studies concerning ICDAS repeatability usually present kappa coefficients for ordinal scale. ${ }^{22}$ On the other hand, Rodrigues et al. ${ }^{11}$ and Diniz et al. ${ }^{12}$ showed lower unweighted kappa values in their study for both intra- ( 0.61 and 0.59 , respectively) and interexaminer $(0.51)$ repeatability.
The most challenging aspect of caries detection is the detection of the first changes in the dental surface. ${ }^{7,9}$ The data presented in this article showed a significant decrease in ICDAS scores from before to after the e-learning program. This could be explained by the interactive online training, which allowed the examiners to visually differentiate the ICDAS scores, especially among codes 1,2 , and 3 and between scores 5 and 6 . It can be seen that before the e-learning program training, the number of sites classified as codes $1,2,3,5$, and 6 were greater than after the e-learning. The fact that examiners were first introduced to the ICDAS criteria based on a published study, which presented a brief description of the ICDAS codes with no image examples, when compared to the e-learning program, probably can explain these results.

The histological assessment used in this study to validate caries extension according to demineralization depth was based on the Ekstrand et al. ${ }^{4}$ classification. In this study the tooth hemisection was carefully conducted through the center of the test site to be confident that the deepest aspect of the lesion was achieved. In previous studies comparing the visual criterion and histological classification, the Spearman correlation between them has been strong with values varying from 0.87 to $0.93 .{ }^{4,23}$ Ekstrand et al. ${ }^{9}$ found strong correlation ( 0.91 to 0.96 ) between ICDAS and histological depth of the lesion. In our study, the correlation was not as strong, with values varying from 0.57 to 0.61 for before and after the e-learning program, respectively. Jablonski-Momeni et al. ${ }^{10}$ presented low correlation coefficient $(0.43$ to 0.68 ) as well using the same histological classification proposed by Ekstrand et al. ${ }^{4}$

The area under the ROC curve $\left(A_{z}\right)$ showed good accuracy of the ICDAS in phases I and II ( 0.80 and 0.88 , respectively) to detect occlusal caries lesions. Other studies had shown that the ICDAS produced areas under the ROC curves from 0.73 to $0.85^{10}$ and $0.75,{ }^{11}$ which agrees with our results. The ROC curve shows the tradeoff between sensitivity and specificity (any increase in sensitivity will be accompanied by a decrease in specificity). The closer the curve follows the left-hand border and then the top border of the ROC space, the higher the overall accuracy of the test. ${ }^{24}$

In this study, before the e-learning training the specificity was low (0.36) and sensitivity was high (0.92). This means that a careful detection is required to avoid false-positive interpretations, considering that large numbers of sound sites were erroneously 
scored as carious and those sites could be given preventive as well as operative intervention in clinical practice, being a benefit or a detriment for patients. According to Jablonski-Momeni et al., ${ }^{10}$ who also found low specificity $(0.52)$ for ICDAS, this could be explained due to stained sites, areas of fluorosis, or developmental defects being incorrectly scored as caries. In our study, it was possible to observe that the students had difficulty distinguishing caries from enamel defects and stains. On the other hand, after the e-learning training, specificity was significantly improved (0.77), and sensitivity was slightly reduced (0.87). These results showed that, after the online training, the students were better able to differentiate fluorosis, stain, and hypoplasia from sound surfaces. Although we can observe a reduction in terms of sensitivity, it is important to point out that there is a great balance between specificity and sensitivity after the e-learning training. The results presented in this article show that the ICDAS e-learning program could assist in the detection of occlusal caries lesions and clearly define the ICDAS codes, being useful and easy to understand. Other effective modalities to convey instructions about the ICDAS criteria, such as a single lecture of ninety minutes and hands-on training session, also demonstrated reasonable learning experience of this methodology. ${ }^{25}$

According to Autio-Gold and Tomar, ${ }^{26}$ students' attitudes toward caries prevention can impact receptivity to training and subsequent involvement in preventive services they would provide in their future practices. In that survey, the authors evaluated dental students' opinions and knowledge regarding the progression of enamel caries. It was observed that the results were conflicting, as the students were almost equally divided on whether or not most incipient lesions will progress into cavities. In our study, it was possible to realize that the dental students had a positive reaction concerning the ICDAS training-learning program. In addition, they were very receptive to this new visual criterion, and it could be considered an improvement in their diagnostic skill. Given that the dental students continued with their clinical activities during the six-week interval in which this study was completed, the improvements observed in their diagnostic skills are likely to have occurred from a combination of the participation in the study, the ICDAS e-learning program, and their ongoing clinical training and knowledge acquisition outside of the study setting. It is important to point out that more training and practice should be offered to the students during their dental education, with an objective to help them develop skills concerning caries detection.

In conclusion, the ICDAS e-learning program improved the reproducibility and validity in terms of specificity of the diagnostic skills of the students for the detection of occlusal caries lesions in permanent teeth. It could be suggested that the ICDAS e-learning program can be a helpful tool for providing feedback to students on caries detection protocol.

\section{REFERENCES}

1. Pitts NB, Longbottom C. Preventive care advised (PCA)/ operative care advised (OCA): categorizing caries by the management option. Community Dent Oral Epidemiol 1995;23:55-9.

2. Pitts NB. Diagnostic tools and measurements: impact on appropriate care. Community Dent Oral Epidemiol 1997;25:24-35.

3. Pitts NB. Review of the ICW-CCT meeting: the importance of early detection and the philosophy/approach of ICDAS (International Caries Detection \& Assessment System). In: Stookey GK. Proceedings of the 6th Indiana Conference Indiana University School of Dentistry: early detection of dental caries III. Cincinnati: SpringDot, 2003.

4. Ekstrand KR, Ricketts DN, Kidd EA. Reproducibility and accuracy of three methods for assessment of demineralization depth on the occlusal surface: an in vitro examination. Caries Res 1997;31:224-31.

5. Nyvad B, Machiulskiene V, Baelum V. Reliability of a new caries diagnostic system differentiating between active and inactive caries lesions. Caries Res 1999;33:252-60.

6. Bader JD, Shugars DA, Bonito AJ. Systematic reviews of selected dental caries diagnostic and management methods. J Dent Educ 2001;65(10):960-8.

7. Ismail AI, Sohn W, Tellez M, Amaya A, Sen A, Hasson $\mathrm{H}$, Pitts NB. The International Caries Detection and Assessment System (ICDAS): an integrated system for measuring dental caries. Community Dent Oral Epidemiol 2007;35:170-8.

8. Zandoná AF, Zero DT. Diagnostic tools for early caries detection. J Am Dent Assoc 2006;137:1675-84.

9. Ekstrand KR, Martignon S, Ricketts DJN, Qvist V. Detection and activity assessment of primary coronal caries lesions: a methodologic study. Oper Dent 2007;32:225-35.

10. Jablonski-Momeni A, Stachniss V, Ricketts DN, HeinzelGutenbrunner M, Pieper K. Reproducibility and accuracy of the ICDAS-II for detection of occlusal caries in vitro. Caries Res 2008;42:79-87.

11. Rodrigues JA, Hug I, Diniz MB, Lussi A. Performance of fluorescence methods, radiographic examination, and ICDAS II on occlusal surfaces in vitro. Caries Res 2008;42:297-304.

12. Diniz MB, Rodrigues JA, Hug I, Cordeiro RCL, Lussi A. Reproducibility and accuracy of the ICDAS-II for occlusal caries detection. Community Dent Oral Epidemiol 2009;37(5):399-404.

13. Finlayson TL, Siefert K, Ismail AI, Sohn W. Psychosocial factors and early childhood caries among low-income 
African American children in Detroit. Community Dent Oral Epidemiol 2007;35:439-48.

14. Sohn W, Ismail A, Amaya A, Lepkowski J. Determinants of dental care visits among low-income African American children. J Am Dent Assoc 2007;138:309-18.

15. Kühnisch J, Berger S, Goddon I, Senkel H, Pitts N, Heinrich-Weltzien R. Occlusal caries detection in permanent molars according to WHO basic methods, ICDAS II and laser fluorescence measurements. Community Dent Oral Epidemiol 2008;36(6):475-84.

16. Metz CE. Basic principles of ROC analysis. Sem Nuc Med 1978;8:283-98.

17. Adeyemi AA, Jarad FD, Komarov GN, Pender N, Higham SM. Assessing caries removal by undergraduate dental students using quantitative light-induced fluorescence. J Dent Educ 2008;72(11):1318-23.

18. Selwitz RH, Ismail A, Pitts NB. Dental caries. Lancet 2007;369:51-9.

19. Nyvad B, Machiulskiene V, Baelum V. Construct and predictive validity of clinical caries diagnostic criteria assessing lesion activity. J Dent Res 2003;82:117-22.

20. Park HM, Jung HW. Evaluating interrater agreement with intraclass correlation coefficient in SPICE-based software process assessment. Proceedings of the 3rd International Conference on Quality Software (QSIC '03). Washington, DC: IEEE Computer Society, 2003:1-6.
21. Ongkosuwito EM, Dieleman MMJ, Kuijpers-Jagtman AM, Mulder PGH, van Neck JW. Linear mandibular measurements: comparison between orthopantomograms and lateral cephalograms. Cleft Palate Craniofac J 2009;46:147-53.

22. Cohen J. Weighted kappa: nominal scale agreement with provision for scaled disagreement or partial credit. Psycol Bull 1968;70:213-20.

23. Ekstrand KR, Kuzmina I, Bjornadal L, Thylstrup A. Relationship between external and histologic features of progressive stages of caries in the occlusal fossa. Caries Res 1995;29:243-50.

24. Zweig MH, Campbell G. Receiver-operating characteristic (ROC) plots: a fundamental evaluation tool in clinical medicine. Clin Chem 1993;39:561-77.

25. Zandona AGF, Al-Shiha S, Eggertsson H, Eckert G. Student versus faculty performance using a new visual criteria for the detection of caries on occlusal surfaces: an in vitro examination with histological validation. Oper Dent 2009;34:598-604

26. Autio-Gold JT, Tomar SL. Dental students' opinions and knowledge about caries management and prevention. J Dent Educ 2008;72(1):26-32. 